\title{
GIẢI PHÁP THÀNH LẬP BẢN ĐỒ ĐỊA HÌNH TỪ DŨ̉ LIỆU UAV VÙNG CÓ PHỦ THỰC VẠTT
}

\author{
HOÀNG TH! THỦY, ĐINH CÔNG HÒA
}

Đại họ Mỏ-Địa chất

\section{Tóm tắt:}

Việc ưng dụng công nghệ bay chup bằng máy bay không người lái trong công tác xây dưng mô hình số bề mặt và bản đồ địa hình đã được thực hiện trong một số nghiên cúu. Tuy vậy, chura có nghiên cứu đầy đủ và hệ thống về độ chính xác và giải pháp nâng cao độ chính xác, đặc biệt khi địa hình có thực vật che phủ. Trong nội dung nghiên cưu này, tác giả đề xuất giải pháp hiệu chỉnh mô hình phủ thực vật (DSM) đối với dũ liệu ảnh máy bay không người lái (UAV), dựa vào các điểm kiểm tra đo bằng công nghệ GPS động RTK (CORS) trong thành lập bản đồ địa hình. Kết quả nghiên cứu là phần mềm tự động hiệu chỉnh độ cao điểm đo ảnh trên bề mặt lớp phủ thực vật về độ cao điểm địa hình. Số liệu thực nghiệm đã minh chưng tính chính xác của thuật toán.

\section{Mở đầu}

Những năm gần đây, trước những đòi hỏi của nhu cầu thực tiễn của đời sống kinh tế xã hội và an ninh quốc phòng đã xuất hiện những mô hình máy bay không người lái. Việc ứng dụng công nghệ bay chụp UAV trong công tác xây dựng mô hình số bề mặt (Digital Surface Model - DSM) và bản đồ địa hình đã được thực hiện trong một số nghiên cứu. Máy bay không người lái MD-1000 đã được Xí nghiệp bay chụp ảnh hàng không - Cục bản đồ Bộ tổng tham mưu sử dụng trong công tác thành lập bản đồ 3D cho khu vực Mỹ Đình và Thái Nguyên. Năm 2014, các tác giả Vũ Phan Long \& Lê Thắng thông qua kết quả thử nghiệm ứng dụng kết hợp giữa hệ thống UAV Swinglet CAM (Sensfly - Thụy sỹ) và UX5 (Trimble -Mỹ) trong thành lập bản đồ 3D hành lang tuyến điện, đã đưa ra khẳng định rằng: UAV hoàn toàn có thể sử dụng trong giám sát phạm vi hẹp, lập bản đồ $3 \mathrm{D}$ và thành lập bản đồ địa hình 1:2000 [6]. Năm 2014, các tác giả Võ Chí Mỹ và Đào Ngọc Long đã thử nghiệm thành công ứng dụng máy bay không người lái trong đo vẽ bản đồ tỷ lệ lớn và giám sát các thành phần tài nguyên và môi trường [5]. Trong một nghiên cứu năm 2016, tác giả Bùi Tiến Diệu và đồng nghiệp đã xây dựng quy trình công nghệ sử dụng ảnh máy bay không người lái, xây dựng các sản phẩm bản đồ (mô hình số bề mặt, mô hình số độ cao, bản đồ trực ảnh, bản đồ 3D) [8]. Các tác giả Bùi Ngọc Quý và Phạm Văn Hiệp cũng đã thành lập bản đồ 3D bằng công nghệ UAV khu vực đồng bằng [7]. Tác giả Mai Văn Sỹ và cộng sự đã nghiên cứu thành lập bản đồ tỷ lệ lớn từ dữ liệu ảnh bay chụp UAV [9]. Trong một nghiên cứu khác của tác giả Bùi Tiến Diệu và các cộng sự năm 2017 , thiết bị UAV được sử dụng bay chụp xây dựng mô hình số bề mặt khu vực bờ dốc [10]. Tuy nhiên, các nghiên cứu này chỉ giới hạn trong khu vực có điều kiện địa hình đơn giản và bằng phẳng. Bên cạnh các đánh giá về độ chính xác khi thay đổi đồ hình và số điểm khống chế ảnh, chưa đưa ra được một quy trình bay chụp đầy đủ và rõ ràng cho các đặc điểm địa hình khác nhau. Khả năng ứng dụng UAV cần được đánh giá chi tiết và toàn diện.

Trong lĩnh vực thành lập bản đồ địa hình tỷ lệ lớn, chưa có nghiên cứu về độ chính xác và giải pháp nâng cao độ chính xác, đặc biệt là khi địa hình có lớp thực vật che phủ. Có thể nhận thấy rằng: số lượng nghiên cứu về ứng dụng công nghệ UAV kết hợp với công nghệ CORS ở Việt Nam còn hạn chế, các nghiên cứu cũng chưa đưa ra được giải pháp kỹ thuật cụ thể về quy trình và các tiêu

\footnotetext{
Ngày nhận bài: 05/05/2020, ngày chuyển phản biện: 09/05/2020, ngày chấp nhận phản biện: 15/05/2020, ngày chấp nhận đăng: 18/05/2020
} 
chuẩn kỹ thuật thành lập được bản đồ tỷ lệ lớn từ công nghệ CORS và UAV.

Trong nội dung nghiên cứu này, tác giả đề xuất giải pháp hiệu chỉnh mô hình phủ thực vật đối với dữ liệu ảnh UAV dựa vào các điểm đo kiểm tra bằng công nghệ CORS trong thành lập bản đồ địa hình.

\section{Giải pháp hiệu chỉnh mô hình số bề mặt vùng có phủ thực vật từ dữ liệu UAV trong thành lập bản đồ địa hình tỷ lệ lớn}

\subsection{Nội dung bài toán}

Trong quá trình bay chụp bằng công nghệ UAV, đối với khu vực có phủ thực vật che khuất, chúng ta chỉ nhận được dữ liệu của bề mặt phủ thực vật. Nhiệm vụ bài toán đặt ra là hiệu chỉnh dữ liệu bề mặt phủ thực vật về dữ liệu bề mặt địa hình tại các vị trí tương ứng. Như vậy, nếu chúng ta xác định được mô hình phủ thực vật tại khu vực bay chụp thì sẽ xác định được độ cao bề mặt địa hình. Để giải quyết bài toán này, cần chia nhỏ khu đo tại từng điểm đo ảnh với bán kính tùy ý $(20 \mathrm{~m}, 50 \mathrm{~m}$, $100 \mathrm{~m}, . .$.$) để xác định mô hình phủ thực vật tại đó, dựa vào chiều cao của lớp phủ thực vật hoặc độ$ cao điểm địa hình, với số điểm vừa đủ. Độ cao điểm địa hình (điểm kiểm tra) được xác định bằng công nghệ CORS hoặc máy toàn đạc điện tử.

Để hiệu chỉnh độ cao điểm đo ảnh bằng công nghệ UAV, tại mỗi điểm cần xác định hàm biểu diễn lớp phủ thực vật đối với bề mặt địa hình có dạng:

$$
\mathrm{Z}_{\mathrm{i}}=\mathrm{F}\left(\mathrm{x}_{\mathrm{i}}, \mathrm{y}_{\mathrm{i}}\right)
$$

trong đó: $\mathrm{Z}_{\mathrm{i}}$ là độ dày lớp phủ thực vật tại vị trí tọa độ $\left(\mathrm{x}_{\mathrm{i}}, \mathrm{y}_{\mathrm{i}}\right)$

Tùy theo từng khu vực địa hình, hàm $\mathrm{F}$ được biểu diễn dưới dạng đa thức bậc 1 , bậc 2 hoặc bậc 3. Cụ thể:

Hàm đa thức bậc 1: $\mathrm{Z}=\mathrm{Z}_{0}+\mathrm{Ax}+\mathrm{By}$

Hàm đa thức bậc 2: $\mathrm{Z}=\mathrm{Z}_{0}+\mathrm{Ax}+\mathrm{By}+\mathrm{Cx}^{2}+\mathrm{Dy}^{2}+\mathrm{Exy}$

Hàm đa thức bậc 3: $\mathrm{Z}=\mathrm{Z}_{0}+\mathrm{Ax}+\mathrm{By}+\mathrm{Cx}^{2}+\mathrm{Dy}^{2}+\mathrm{Exy}+\mathrm{Fx}^{3}+\mathrm{Gy}^{3}+\mathrm{Hx}^{2} \mathrm{y}+\mathrm{KXY}{ }^{2}$

Để xác định các hệ số của hàm đa thức đối với từng điểm đo ảnh số $U A V$, cần thiết phải sử dụng các điểm kiểm tra. Số lượng điểm kiểm tra nằm trong khoảng 3-10 tùy theo số bậc của hàm số. Khi số điểm kiểm tra lớn hơn số các hệ số, có thể áp dụng nguyên lý số bình phương nhỏ nhất để xác định giá trị xác xuất nhất của các hệ số. Phương trình số hiệu chỉnh các hệ số tương ứng các bậc của hàm số (2) như sau:

$$
\begin{aligned}
& \mathrm{V}_{\mathrm{z}}=1 \cdot \mathrm{dZ}_{0}+\mathrm{x} \cdot \mathrm{dA}+\mathrm{y} \cdot \mathrm{dB}+\mathrm{l}_{\mathrm{z}} ; \mathrm{l}_{\mathrm{z}}=-\mathrm{Z} \\
& \mathrm{V}_{\mathrm{z}}=1 \cdot \mathrm{dZ} \mathrm{Z}_{0}+\mathrm{x} \cdot \mathrm{dA}+\mathrm{y} \cdot \mathrm{dB}+\mathrm{x}^{2} \cdot \mathrm{dC}+\mathrm{y}^{2} \cdot \mathrm{dD}+\mathrm{xy} \cdot \mathrm{dE}+\mathrm{l}_{\mathrm{z}} ; \mathrm{l}_{\mathrm{z}}=-\mathrm{Z} \\
& \mathrm{V}_{\mathrm{z}}=1 \cdot \mathrm{dZ}_{0}+\mathrm{x} \cdot \mathrm{dA}+\mathrm{y} \cdot \mathrm{dB}+\mathrm{x}^{2} \cdot \mathrm{dC}+\mathrm{y}^{2} \cdot \mathrm{dD}+\mathrm{xy} \cdot \mathrm{dE}+\mathrm{x}^{3} \cdot \mathrm{d} \mathrm{l}_{\mathrm{z}} ; \mathrm{l}_{\mathrm{z}}=-\mathrm{Z}
\end{aligned}
$$

Bằng bài toán bình sai gián tiếp, xác định được các hệ số của mô hình (3), từ đó hiệu chỉnh độ cao bề mặt phủ thực vật về độ cao bề mặt địa hình (4):

Ma trận hệ phương trình sai số: $\mathrm{V}=\mathrm{AX}+\mathrm{L}$;

Ma trận hệ phương trình chuẩn: $\quad \mathrm{A}^{\mathrm{T}} \mathrm{A} \cdot \mathrm{X}+\mathrm{A}^{\mathrm{T}} \mathrm{A} \cdot \mathrm{L}=0$; hoặc $\mathrm{NX}+\mathrm{B}=0$ 
Véc tơ ẩn số

$$
\mathrm{X}=-\mathrm{N}^{-1} \mathrm{~B}
$$

Hiệu chỉnh độ cao phủ thực vật về bề mặt địa hình (5): $\quad \mathrm{H}_{\text {đh }}=\mathrm{H}_{\mathrm{DSM}}-\mathrm{Z}$

\subsection{Quy trình thục hiện hiệu chỉnh}

Mật độ điểm ảnh UAV trong quá trình bay chụp rất lớn, khoảng cách các điểm từ $1 \mathrm{~m}$ đến $2 \mathrm{~m}$, trong khi đó, điểm kiểm tra được phân bố trên toàn bộ khu vực, tại các điểm đặc trưng của lớp phủ thực vật. Tại từng điểm đo ảnh, tăng dần bán kính khoanh vùng để xác định vị trí các điểm kiểm tra gần nhất. Khi số điểm kiểm tra vừa đủ tùy theo hàm số đa thức đã chọn, sẽ xây dựng mô hình bề mặt phủ thực vật tại đó và tính số hiệu chỉnh. Để thực hiện được bài toán này, một modul chương trình cho việc tự động hóa công tác tính toán đã được xây dựng nhằm đảm bảo tính chính xác và hiệu quả kinh tế. Giao diện chương trình được thể hiện trên hình 1 và sơ đồ khối chương trình nêu trong hình 2 .

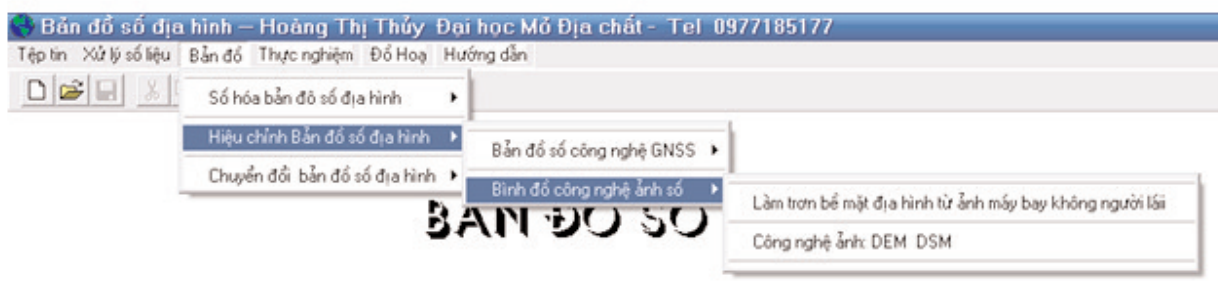

Hình 1: Giao diện modul chwơng trình hiệu chỉnh độ cao điểm ảnh UAV

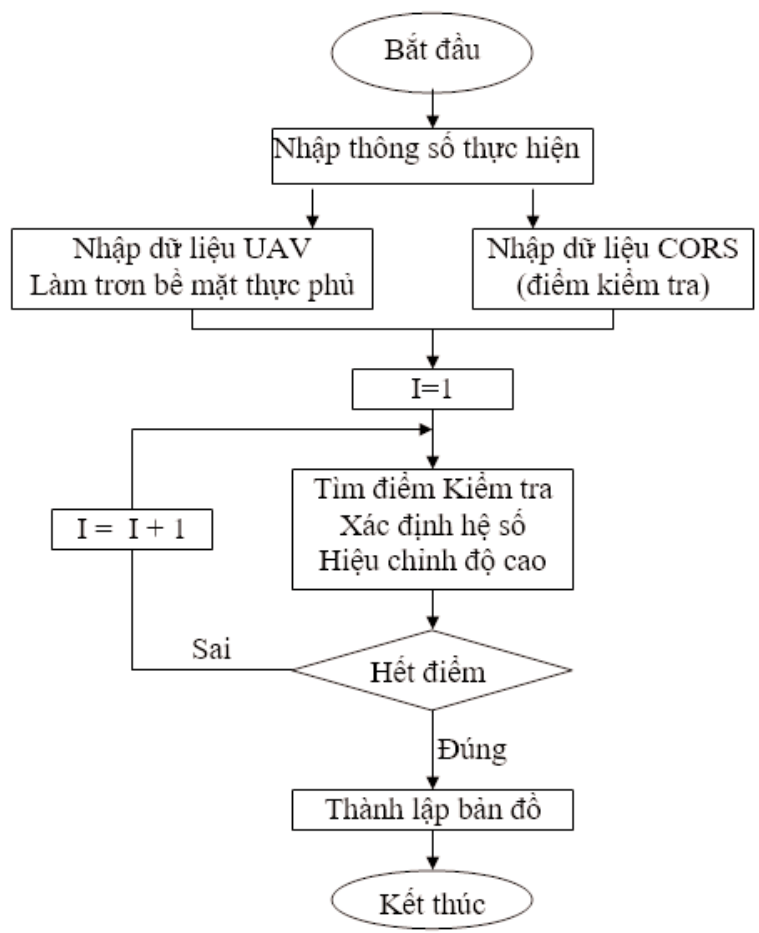

Hình 2: So đồ khối thục hiện của chuơng trình

\section{Kết quả thực nghiệm}

\subsection{Số liệu mô hình}


Để kiểm định kết quả tính toán, chương trình thực nghiệm được tiến hành trên khu vực có diện tích $1 \mathrm{~km}^{2}$ (hình $3 \mathrm{a}$ ). Bằng thuật toán mô hình số, xây dựng mô hình phủ thực vật bậc 3 (hình 3b) đã xây dựng được mô hình số bề mặt (DSM) (hình 3c). Trên bản đồ gốc, 30 trong số 1087 điểm đã được lựa chọn bảo đảm phủ đều trên khu đo làm điểm kiểm tra, kết quả của quá trình hiệu chỉnh dựa vào điểm kiểm tra sẽ nhận được mô hình số địa hình (hình $3 \mathrm{~d}$ ). Kết quả đánh giá độ chính xác hiệu chỉnh độ cao địa hình được thể hiện trong bảng 1 . Kết quả thực nghiệm cho thấy rằng: cơ sở lý thuyết và modul chương trình là phù hợp và có độ tin cậy cao. Sai số độ cao các điểm so với trị thực là $\mathrm{m}= \pm 0,2$ mét.

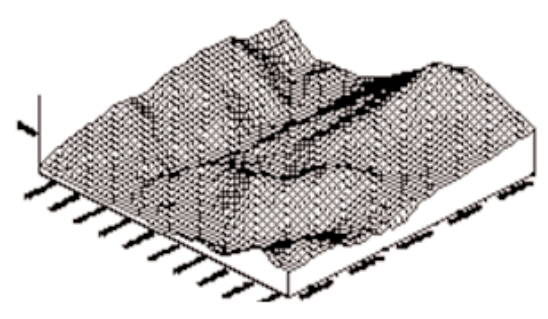

Hình 3a: Mô hình số liệu gốc

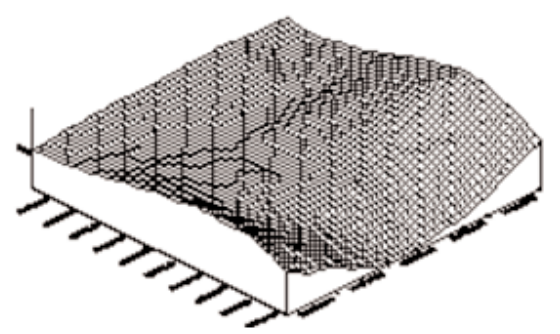

Hình 3c: Mô hình số liệu DSM

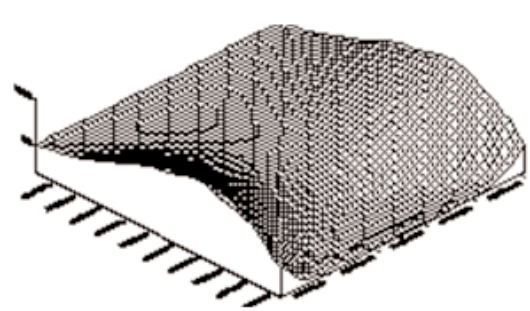

Hìn 3b: Mô hìh phủ thục vật

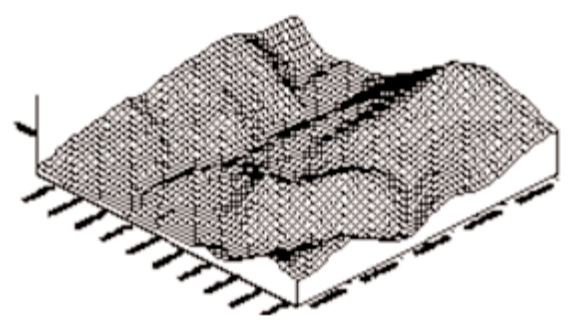

Hình 3d: Mô hình số liệu DEM

\begin{tabular}{|c|c|c|c|c|c|c|}
\hline \multicolumn{7}{|c|}{ KÉT QUÁ ĐÁNH GIÁ ĐỌ CHÍNH XÁC HIẸU CHİNH ĐO CAO ĐİA HìNH } \\
\hline No & $\mathrm{X}$ & $\mathrm{Y}$ & $\mathrm{H}$ (thực) & H1(hiệuchinh) & $\mathrm{d}=\mathrm{H}-\mathrm{H} 1$ & $\mathrm{dd}$ \\
\hline 1 & 2348165.338 & 472156.141 & 75.00 & 75.00 & 0.00 & 0.0000 \\
\hline 2 & 2348373.609 & 472157.908 & 55.00 & 54.99 & -0.01 & 0.00010 \\
\hline$\ldots \ldots$ & & & & & & \\
\hline 1086 & 2348414.026 & 472506.895 & 90.00 & 90.00 & 0.00 & 0.0000 \\
\hline 1087 & 2348390.707 & 472492.209 & 90.00 & 90.00 & 0.00 & 0.0000 \\
\hline$[\mathrm{dd}]=92.1218451486419$ & $\mathrm{n}=1087$ & $\mathrm{~m}= \pm \sqrt{\frac{[d d]}{n}}= \pm 0.291 \mathrm{~m}$ & $\mathrm{~m}= \pm \sqrt{\frac{[d d]}{2 n}}= \pm 0.206 \mathrm{~m}$ \\
\hline
\end{tabular}

Bảng 1: Kết quả đánh giá độ chính xác: Mô hình thực nghiệm

\subsection{Số liệu thục tế}

Để kiểm chứng kết quả nghiên cứu, chương trình thực nghiệm đã được tiến hành trên số liệu thực tế tại TP Lạng Sơn. Trên cơ sở bản đồ địa hình tỷ lệ 1:1.000 thành lập từ số liệu đo trực tiếp bằng phương pháp toàn đạc điện tử và mô hình số được thành lập bằng công nghệ UAV, tiến hành so sánh kết quả nhận được. Hình $4(\mathrm{a}, \mathrm{b}, \mathrm{c})$ là mô hình $3 \mathrm{D}$ của kết quả đo trực tiếp, mô hình DEM và DSM nhận được từ dữ liệu UAV 


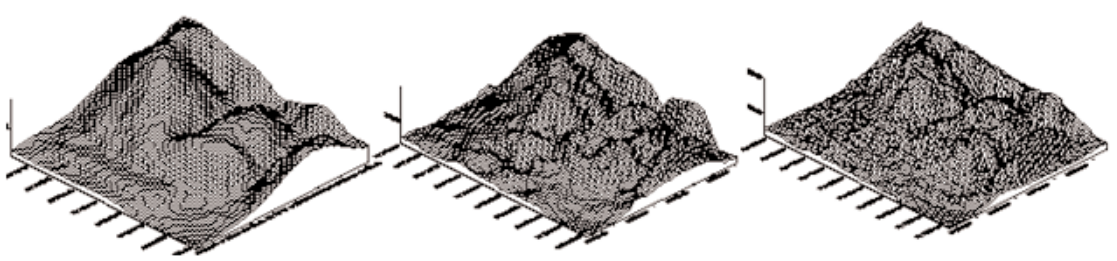

Hình 4a: Mô hình sl đo trục tiếp Hình 4b: Mô hình DEM Hình 4c: Mô hình DSM

Để khảo sát kết quả nghiên cứu, tiến hành đo các điểm kiểm tra bằng công nghệ CORS, thông qua modul hiệu chỉnh độ cao. Kết quả này được so sánh với bản đồ đo trực tiếp và so sánh giữa 2 mô hình với nhau được thành lập bằng công nghệ ảnh số. Kết quả được thể hiện trong bảng 2 , bảng 3 và bảng 4 . Từ kết quả thực nghiệm, cho thấy rằng:

- Độ chính xác của mô hình càng cao khi số điểm kiểm tra càng lớn; Từ 350 đến 1000 điểm kiểm tra trong tổng số 35.000 điểm đo (tính ra được tỷ lệ $1: 100$ ).

- Dữ liệu mô hình số bề mặt (DSM) từ công nghệ ảnh số UAV cần phải làm trơn bằng hàm đa thức bậc 2 hoặc bậc 3 với bán kính từ $20 \mathrm{~m}$ đến $100 \mathrm{~m}$, tùy thuộc vào bề mặt lớp phủ thực vật, khi đó kết quả hiệu chỉnh độ cao từ dữ liệu ảnh số sẽ đạt độ chính xác khi thành lập bản đồ số địa hình tỷ lệ lớn.

\begin{tabular}{|c|c|c|c|c|c|c|}
\hline \multirow{2}{*}{$\begin{array}{c}\text { Bán } \\
\text { kính làm } \\
\text { trơn }(\mathrm{m})\end{array}$} & \multicolumn{5}{|c|}{ KÉT QUẢ KHÅO SÁT MÔ HİNH DEM } \\
\cline { 2 - 7 } & $\mathrm{m}= \pm \sqrt{\frac{[d d]}{n}}$ & $\mathrm{~m}= \pm \sqrt{\frac{d d d]}{2 n}}$ & $\mathrm{~m}= \pm \sqrt{\frac{[d d]}{n}}$ & $\mathrm{~m}= \pm \sqrt{\sqrt{\frac{d d d]}{2 n}}}$ & $\mathrm{~m}= \pm \sqrt{\frac{d d d]}{n}}$ & $\mathrm{~m}= \pm \sqrt{\frac{[d d]}{2 n}}$ \\
\hline $\mathrm{R}=0$ & $2.85777 \mathrm{~m}$ & $2.02075 \mathrm{~m}$ & $2.10068 \mathrm{~m}$ & $1.48540 \mathrm{~m}$ & $0.34218 \mathrm{~m}$ & $0.24196 \mathrm{~m}$ \\
\hline $\mathrm{R}=20$ & $2.61929 \mathrm{~m}$ & $1.85212 \mathrm{~m}$ & $1.82988 \mathrm{~m}$ & $1.29392 \mathrm{~m}$ & $0.27695 \mathrm{~m}$ & $0.19583 \mathrm{~m}$ \\
\hline $\mathrm{R}=50$ & $1.70805 \mathrm{~m}$ & $1.20778 \mathrm{~m}$ & $1.25556 \mathrm{~m}$ & $0.88781 \mathrm{~m}$ & $0.24959 \mathrm{~m}$ & $0.17649 \mathrm{~m}$ \\
\hline $\mathrm{R}=70$ & $1.84938 \mathrm{~m}$ & $1.30771 \mathrm{~m}$ & $1.29632 \mathrm{~m}$ & $0.91663 \mathrm{~m}$ & $0.25669 \mathrm{~m}$ & $0.18151 \mathrm{~m}$ \\
\hline $\mathrm{R}=100$ & $2.01114 \mathrm{~m}$ & $1.42209 \mathrm{~m}$ & $1.28005 \mathrm{~m}$ & $0.90513 \mathrm{~m}$ & $0.25543 \mathrm{~m}$ & $0.18062 \mathrm{~m}$ \\
\hline
\end{tabular}

Bảng 2: Kết quả đánh giá độ chính xác: Mô hình số địa hình

\begin{tabular}{|c|c|c|c|c|c|c|}
\hline \multirow{3}{*}{$\begin{array}{l}\text { Bán kính } \\
\text { làm trơn } \\
\text { (m) }\end{array}$} & \multicolumn{6}{|c|}{ KÊT QUẢ KHẢO SÁT MÔ HİNH DSM } \\
\hline & \multicolumn{2}{|c|}{150 điểm kiểm tra } & \multicolumn{2}{|c|}{350 điểm kiểm tra } & \multicolumn{2}{|c|}{1000 điểm kiểm tra } \\
\hline & $\mathrm{m}= \pm \sqrt{\frac{[d d]}{n}}$ & $\mathrm{~m}= \pm \sqrt{\frac{[d d]}{2 n}}$ & $\mathrm{~m}= \pm \sqrt{\frac{[d d]}{n}}$ & $\mathrm{~m}= \pm \sqrt{\frac{[d d]}{2 n}}$ & $\mathrm{~m}= \pm \sqrt{\frac{d d]}{n}}$ & $\mathrm{~m}= \pm \sqrt{\frac{[d d]}{2 n}}$ \\
\hline $\mathrm{R}=0$ & $4.13728 \mathrm{~m}$ & $2.92550 \mathrm{~m}$ & $3.26195 \mathrm{~m}$ & $2.30655 \mathrm{~m}$ & $0.66301 \mathrm{~m}$ & $0.46882 \mathrm{~m}$ \\
\hline $\mathrm{R}=20$ & $3.06931 \mathrm{~m}$ & $2.17033 \mathrm{~m}$ & $2.10725 \mathrm{~m}$ & $1.49005 \mathrm{~m}$ & $0.31197 \mathrm{~m}$ & $0.22060 \mathrm{~m}$ \\
\hline $\mathrm{R}=50$ & $1.74692 \mathrm{~m}$ & $1.23526 \mathrm{~m}$ & $1.24524 \mathrm{~m}$ & $0.88052 \mathrm{~m}$ & $0.25113 \mathrm{~m}$ & $0.17758 \mathrm{~m}$ \\
\hline $\mathrm{R}=70$ & $1.80750 \mathrm{~m}$ & $1.27810 \mathrm{~m}$ & $1.24958 \mathrm{~m}$ & $0.88359 \mathrm{~m}$ & $0.25331 \mathrm{~m}$ & $0.17912 \mathrm{~m}$ \\
\hline $\mathrm{R}=100$ & $1.91673 \mathrm{~m}$ & $1.35533 \mathrm{~m}$ & $1.24853 \mathrm{~m}$ & $0.88284 \mathrm{~m}$ & $0.24987 \mathrm{~m}$ & $0.17668 \mathrm{~m}$ \\
\hline
\end{tabular}

Bảng 3: Kết quả đánh giá độ chính xác: Mô hình số bề mặt

\begin{tabular}{|c|c|c|c|c|c|}
\hline \multirow{2}{*}{$\begin{array}{c}\text { Điềm } \\
\text { kiềm tra }\end{array}$} & \multicolumn{5}{|c|}{ BÁN KÍNH LA்M TRƠN MÔ HİNH DEM VÁ DSM } \\
\cline { 2 - 6 } & $\mathrm{R}=0 \mathrm{~m}$ & $\mathrm{R}=20 \mathrm{~m}$ & $\mathrm{R}=50 \mathrm{~m}$ & $\mathrm{R}=70 \mathrm{~m}$ & $\mathrm{R}=100 \mathrm{~m}$ \\
\hline 150 điềm & $2.41986 \mathrm{~m}$ & $1.38467 \mathrm{~m}$ & $0.53938 \mathrm{~m}$ & $0.48697 \mathrm{~m}$ & $0.47078 \mathrm{~m}$ \\
\hline 250 điểm & $2.36901 \mathrm{~m}$ & $1.29053 \mathrm{~m}$ & $0.47903 \mathrm{~m}$ & $0.65780 \mathrm{~m}$ & $0.43669 \mathrm{~m}$ \\
\hline 350 điểm & $2.32869 \mathrm{~m}$ & $1.15845 \mathrm{~m}$ & $0.38612 \mathrm{~m}$ & $0.34649 \mathrm{~m}$ & $0.33662 \mathrm{~m}$ \\
\hline 1000 điểm & $1.93228 \mathrm{~m}$ & $0.99312 \mathrm{~m}$ & $0.38182 \mathrm{~m}$ & $0.31917 \mathrm{~m}$ & $0.45482 \mathrm{~m}$ \\
\hline
\end{tabular}

Bảng 4: Kết quả đánh giá độ chính xác giũua 2 mô hình DEM và DSM khi làm trơn 


\section{Kết luận}

Để thực hiện được bài toán này, trên cơ sở lý thuyết bài toán mô hình số dạng đa thức, modul chương trình hỗ trợ quá trình tự động hóa hiệu chỉnh độ cao các điểm đo xác định bằng công nghê ảnh số UAV đã được xây dựng, kết hợp điểm đo kiểm tra bằng công nghệ CORS, đảm bảo tính chính xác và hiệu quả kinh tế.

Kết quả nghiên cứu đã được minh chứng bằng thực nghiệm cho thấy rằng: việc ứng dụng công nghệ ảnh số UAV kết hợp công nghệ CORS trong công tác đo đạc thành lập bản đồ số địa hình tỷ lệ lớn 1.2000 - 1.5000, khoảng cao đều $1 \mathrm{~m}$ đến $2 \mathrm{~m}$, khu vực thực vật che phủ hoàn toàn đảm bảo độ chính xác theo yêu cầu quy phạm hiện hành. Modul chương trình tự động hiệu chỉnh độ cao các điểm xác định bằng công nghê ảnh số UAV khu vực có phủ thực vật che khuất, có ý nghĩa khoa học và thực tiễn. $\bigcirc$

\section{Tài liệu tham khảo}

[1]. Hoàng Ngọc Hà. 2001. Tính toán trắc địa và cơ sở dữ liệu. Nhà xuất bản Giao thông vận tải. Hà Nội

[2]. Đinh Công Hòa. 2011. Lập trình bài toán trắc địa cơ sở. Nhà xuất bản Giao thông vận tải. Hà Nội

[3]. Hoàng Thị Thủy, 2017, Ứng dụng tin học vào bài toán chuyển dổi hệ quy chiếu bản đồ số địa hình, Tạp chí khoa học đo đạc và bản đồ, số 31 .

[4]. Hoàng Thị Thủy, 2017, Nâng cao độ chính xác khi ứng dụng công nghệ RTK trong thành lập bản đồ số tỷ lệ lớn, Tạp chí khoa học đo đạc và bản đồ, số 38 .

[5]. Võ Chí Mỹ, Đào Ngọc Long (2014, Nghiên cứu khả năng ứng dụng máy bay không người lái (UAV) trong công tác đo vẽ bản đồ tỷ lệ lớn và giám sát tài nguyên môi trường. Tuyển tập báo cáo Hội nghị khoa học quốc tế, Viện Khoa học Đo đạc và Bản đồ, Hà Nội.

[6]. Vũ Phan Long, Lê Thắng, Thử nghiệm thiết bị bay không người lái thành lập bản dồ 3D hành lang tuyến điện, Hội nghị khoa học ngành Địa hình quân sự. 2014.

[7]. Bùi Ngọc Quý, Phạm Văn Hiệp, Nghiên cứu xây dựng mô hình 3D từ dữ liệu ảnh máy bay không người lái (UAV). Tạp chí Khoa học kỹ thuật Mỏ - Địa chất, 2017. 4(58): p. 1-11.

[8]. Bùi Tiến Diệu, Nguyễn Cẩm Vân, Hoàng Mạnh Hùng, Nguyễn Quang Minh. Xây dựng mô hình số bề mặt và bản đồ trực ảnh sử dụng công nghệ đo ảnh máy bay không người lái. Hội nghị khoa học: Đo đạc bản đồ với ứng phó biển đổi khí hậu. 2016. Hà Nội.

[9]. Mai Văn Sỹ, Bùi Ngọc Quý, Phạm Văn Hiệp, Lê Đình Quýv, Nghiên cứu xử dụng dữ liệu ảnh máy bay máy bay không người lái (UAV) trong thành lập bản đồ địa hình tỷ lệ lớn. Tạp chí khoa học Đo đạc và bản đồ, 2017. 33.

[10]. Dieu Tien Bui, Nguyen Quoc Long, Bui Xuan Nam, Nguyen Viet Nghia, Pham Van Chung, Le Van Canh, Ngo Thi Phuong Thao, Bui Tien Dung, Bjørn Kristoffersen, Lightweight Unmanned Aerial Vehicle and Structure-from-Motion Photogrammetry for Generating Digital Surface Model for Open-Pit Coal Mine Area and Its Accuracy Assessment. International Conference on Geo-Spatial Technologies and Earth Resources, 2017: p. 17-33.O 


\section{Summary}

\section{UAV - Base data for topographical mapping of vegetation covered areas}

Hoang Thi Thuy, Đinh Cong Hoa, Hanoi University of Mining and Geology

Recentrly, Unmanned Aerial Vehicle (UAV) has been applied popularly in generating Digital Surface Model (DSM) and topographical map. However, there has been not much research to improve accuracy of the generated map, especially when the terrain is covered by vegetation. This study proposed a solution to adjust the UAV derived DSM based on test points measured by GNSSRTK to establishing topographical digital maps. The result is a software that automatically corrects the height of the image measurement point on the surface of plant cover to the terrain elevation. Experimental results have proved the accuracy of the algorithm, the research content can be applied to actual production, achieving economic efficiency. $\bigcirc$

\section{XÂY DỰNG CÔNG CỤ CẢI THIỆN Độ CHÍNH XÁC........}

(Tiếp theo trang 48)

[7]. Rönsdorf, C. (2008). Positional Accuracy Improvement (PAI) Encyclopedia of GIS (pp. 885-891): Springer.

[8]. Tong, X., Liang, D., Xu, G., and Zhang, S. 2011). "Positional accuracy improvement: A comparative study in Shanghai, China".

[9]. Wolf, P., \& Ghilani, C. (2006).
Adjustment Computations Spatial Data Analysis: Hoboken.

[10]. David Siriba (2009). Positional Accuracy Assessment of a Cadastral Dataset based on the Knowledge of the Process Steps used. 12th AGILE International Conference on Geographic Information Science 2009 Leibniz Universität Hannover, Germany. $\bigcirc$

\section{Summary}

\section{Building equipment to improve the precision of old cadastral maps}

Truong Song Hoa, Nguyen Manh Dung - Institute of Geodesy and Cartography

Nguyen Van Hiep - Center for Land Data and Information, General Department of Land Administration

In the article, we introduce solutions to improve the spatial accuracy of the old cadastral maps, which were measured and established before 2009 according to the old regulations. From analyzing the factors affecting the accuracy of the old cadastral map compared to the current requirement, to realize the extent of restriction by each measurement area in each administrative unit of commune/ward, Since then, we have proposed an improved algorithm, a high-precision solution solution for each measurement area and of the commune/ward in general based on the overlapping control points, thereby creating a basis for VPAI software module development. Running on MicroStation has the function of improving spatial accuracy. The improved data will be more consistent with the cadastral data measured in recent years, ensuring the establishment of the land database. The article is the result of a research project at the grassroots level "Research and propose technical solutions to improve the efficiency of the old cadastral map to meet the requirements of building a land database in the current period". $O$ 